\title{
Ideal Wound Closure Methods for Minimizing Scarring After Surgery
}

Rei Ogawa

\section{Contents}

21.1 Background - 186

21.2 Cutaneous Wound Healing and Mechanobiology - 186

21.3 Surgical Techniques that Can Minimize Dermal Tension - 186

21.4 Z-Plasty - 187

21.5 Conclusion -190

References - 191 


\subsection{Background}

The healing of open cutaneous wounds involves the generation of vascularized granulation tissue that closes the gap in the skin. Thereafter, an effective epidermal barrier is created over the granulation tissue. Wounds in which sutures have approximated the edges also undergo granulation, albeit less than in open wounds that must heal by secondary intention. In both cases, the adherence of the wound edges becomes stronger over time: in general, wounded skin acquires $80 \%$ of the dermal strength of the surrounding normal skin by 3 months post-wounding [1].

\subsection{Cutaneous Wound Healing and Mechanobiology}

These wound-healing phenomena are the result of a cascade of complex biochemical events that can be categorized into four general overlapping phases: coagulation, inflammation, proliferation, and remodeling. Significantly, all four phases of wound healing are influenced by both intrinsic and extrinsic mechanical forces. For example, the formation of granulation tissue in the proliferating phase is driven by intrinsic and extrinsic mechanical stimulation of the fibroblasts, myofibroblasts, endothelial cells, and epithelial cells that are in and near the wound. Similarly, myofibroblasts contract the wound in the proliferative phase by mechanical forces that are themselves shaped by many extrinsic forces, including the natural tension in the skin. The remodeling phase can also be influenced by extrinsic mechanical forces [2]. During this phase, fibroblasts secrete collagen and fibronectin, which are key components of the extracellular matrix (ECM). These cells then regulate the volume of the ECM by secreting collagenase. This repeated synthesis and enzymatic breakdown of ECM proteins remodels the three-dimensional structure of the ECM. For proper remodeling, the synthesis and degradation processes must be carefully balanced: if there is too much ECM synthesis, scars can become hypertrophic. Conversely, if there is excessive ECM degradation, the scar can become atrophic. Multiple lines of evidence show that this ECM remodeling process can become deranged by extrinsic forces on the ECM [3]. For example, wounds on the major joints tend to develop hypertro- cyclical tension on the wound. This tension provokes chronic inflammation of the dermis, namely, the unceasing influx and activation of inflammatory cells, the persistent generation of blood vessels and nerve fibers, and the constant production of collagen by the activated fibroblasts. This chronic inflammation blocks the conversion of the granulation tissue into dermis-like tissue by the remodeling process and results in an immature hypertrophic scar that is red, elevated, hard, and painful.

These observations suggest that, to prevent pathological scarring after surgery, it is necessary to ensure that the sutures cause the wound edges to adhere to each other without any tension, even when strong extrinsic forces are placed on the wound. This will allow the granulation tissue to convert smoothly into dermis-like tissue, thereby yielding minimal scarring.

\subsection{Surgical Techniques that Can Minimize Dermal Tension}

The risk of pathological scarring can be greatly reduced by using subcutaneous/fascial tensile reduction sutures [4]. This is because dermal sutures do not effectively reduce tension on the dermis: rather, to achieve this, we must access much deeper structures, namely, the superficial and deep fascia, and suture them. This type of suturing will elevate the wound edges smoothly while placing minimal tension on the dermis. In other words, it will cause the wound edges to attach naturally to each. Only then should dermal and superficial sutures be used. It is very important to realize that dermal sutures on their own cannot reduce the tension on the dermis: this concept is the key to preventing the formation of pathological scars after surgery.

Thus, in the case of benign tumor excision or scar revision surgery in high-tension areas such as the chest wall, the cutaneous mass should be completely excised along with a minimum of normal skin margin and all fatty tissues under the mass. Hence, all tissues above the deep fascia of the muscle are removed. The wound edges are then undermined under the deep fascia, and the deep fascia is sutured with 0 polydioxanone sutures such as PDS ${ }^{\circledR I I}$ (Ethicon, Inc., Somerville, New Jersey). Thereafter, the fibrous membrane in the fatty tissues, namely, the superficial fascia, is sutured using $2-0$ and 3-0 PDS ${ }^{-} I I$. This approach causes the wound edges to adhere naturally to each other. Our previous study 
a

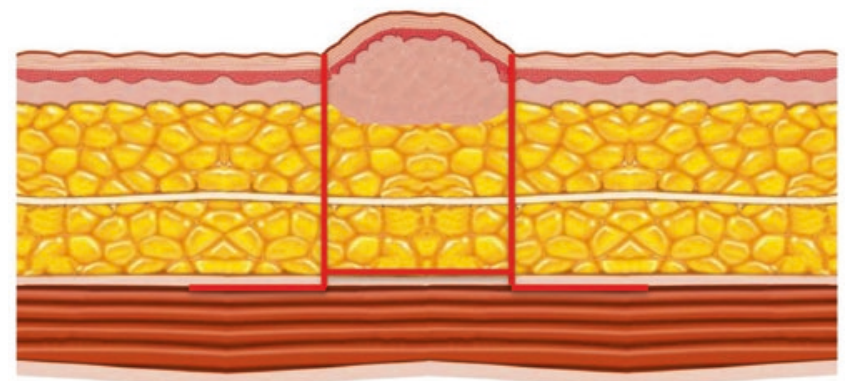

b

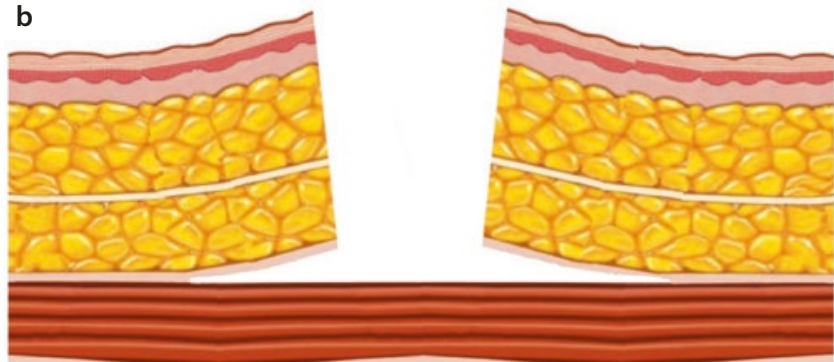

- Fig. 21.1 The ideal closure method for minimizing the tension on the dermis. a The fatty tissues are removed along with the scar or tumor. b Undermining between the deep fascia and the muscle is performed. $\mathbf{c}$ The deep and superficial fasciae are then sutured to release the tension

showed that deep fascia suturing reduced about $90 \%$ of the tension on the wound edge, while superficial fascia suturing reduced the remaining 10\% [5]. After fascial suturing, dermal sutures using 4-0 PDS®II are started. This is followed by superficial sutures with 6-0 polypropylene or nylon sutures such as Proline ${ }^{\circledR}$ or Ethilon ${ }^{\circledR}$ (Ethicon, Inc., Somerville, New Jersey) (• Figs. 21.1, 21.2, and 21.3).

\subsection{Z-Plasty}

Another way to prevent pathological scar formation in high-tension areas is to use zigzag suturing techniques such as the Z-plasty [5]. This is particularly suitable for joint or limb surgery because the fatty tissue layers in these areas are thin: this means that it is difficult to find the superficial fascia and apply the subcutaneous/fascial tensile reduction sutures. Zigzag suturing is also good for long suture wounds because it effectively disrupts the tension on the resulting scar. As a result, zigzag suturing

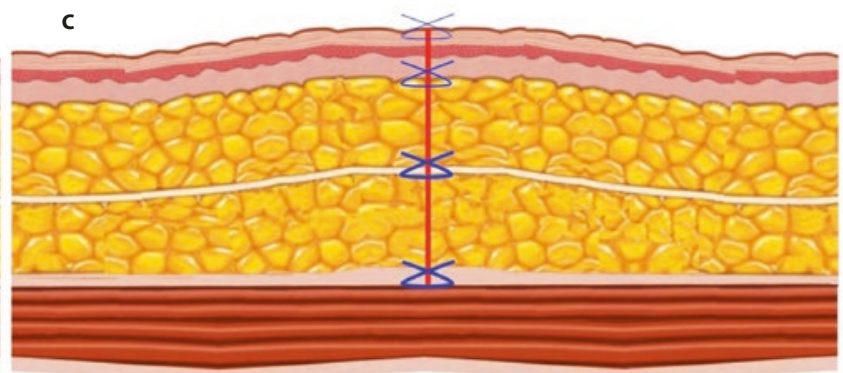

on the dermis. The red lines in a indicate where the tissue is incised and undermined. Dermal sutures themselves do not effectively reduce tension on the dermis. To achieve this, we must access much deeper structures, namely, the superficial and deep fasciae, and suture them

is an effective approach for releasing linear scar contractures. Another major benefit of Z-plasties is that segmented scars mature faster than long linear scars. Thus, if a scar or wound crosses a joint, zigzag incision and suturing will significantly reduce the risk of pathological scar recurrence or development (• Fig. 21.4).

In terms of Z-plasty design, the sides of each triangular flap should be $7-10 \mathrm{~mm}$ long and the pitch between each z-plasty should be $2-4 \mathrm{~cm}$, depending on the total length of the wound [5]. In our experience, this pitch yields the most satisfactory results (personal observations). Dermal sutures can be started after confirming that the triangular flaps are fully elevated and can be transposed with each other.

It should be noted that, because keloids have much stronger inflammation than hypertrophic scars, it is best to use both tension reduction sutures and Z-plasties during keloid revision surgery [5]. This significantly reduces the risk of recurrence (- Fig. 21.5). This risk is also strongly ameliorated by postoperative radiotherapy. 

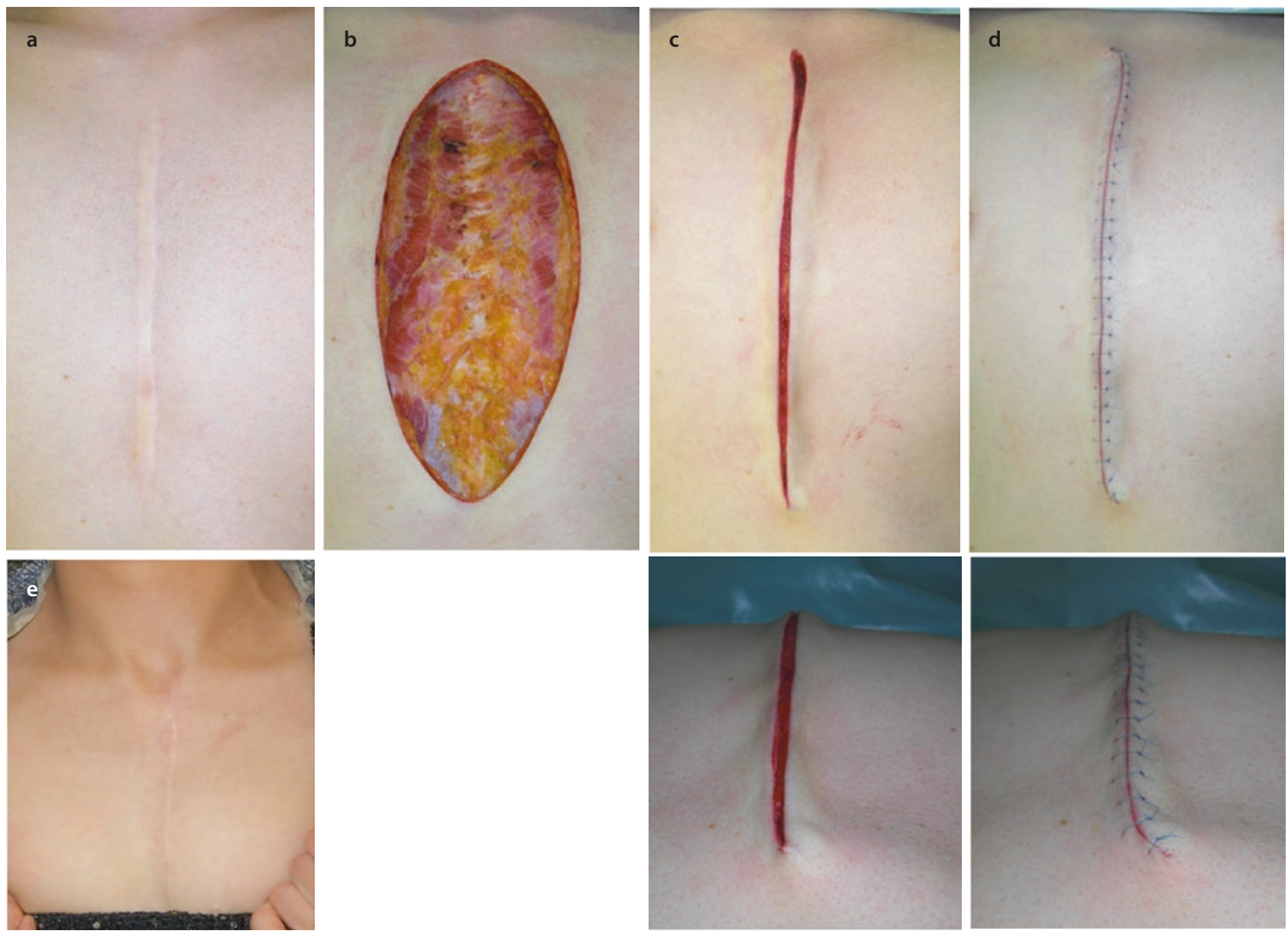

- Fig. 21.2 Chest wall scar revision. a Preoperative appearance of the scar. b View after the scar and fatty tissues were removed. $\mathbf{c}$ View after the deep and superficial fasciae of the pectoralis major muscle were sutured. d View immediately after the dermal sutures and

superficial sutures were placed. e View 2 years after surgery. The wound edges were undermined under the deep fascia, after which the deep fascia was strongly sutured. These deep sutures absorb $90 \%$ of the tension on the wound 

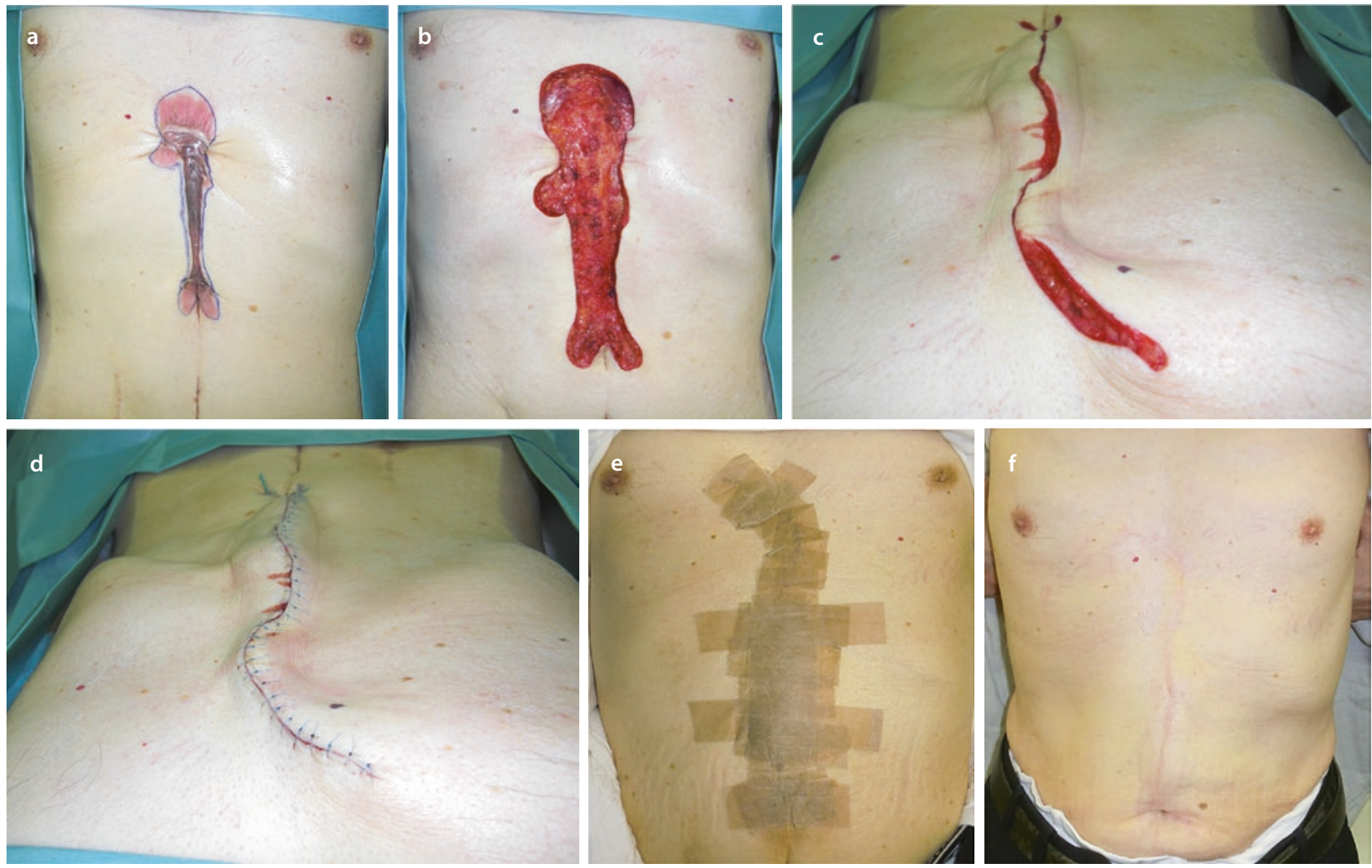

- Fig. 21.3 Abdominal wall scar revision. a Preoperative appearance of the scar and the design of the incisions. b View after the scar and fatty tissues were removed. $\mathbf{c}$ View after the deep and superficial fasciae were sutured. d View immediately after dermal sutures and superficial sutures were placed. e View of the postoperative taping fixation that was used to stabilize the wound and protect it from extrinsic mechanical forces. $\mathbf{f}$ View 2 years after surgery. The anterior sheath of the rectus abdominis muscle was strongly sutured. The wound edges were smoothly elevated so that they attached naturally to each other. Dermal sutures could then be placed
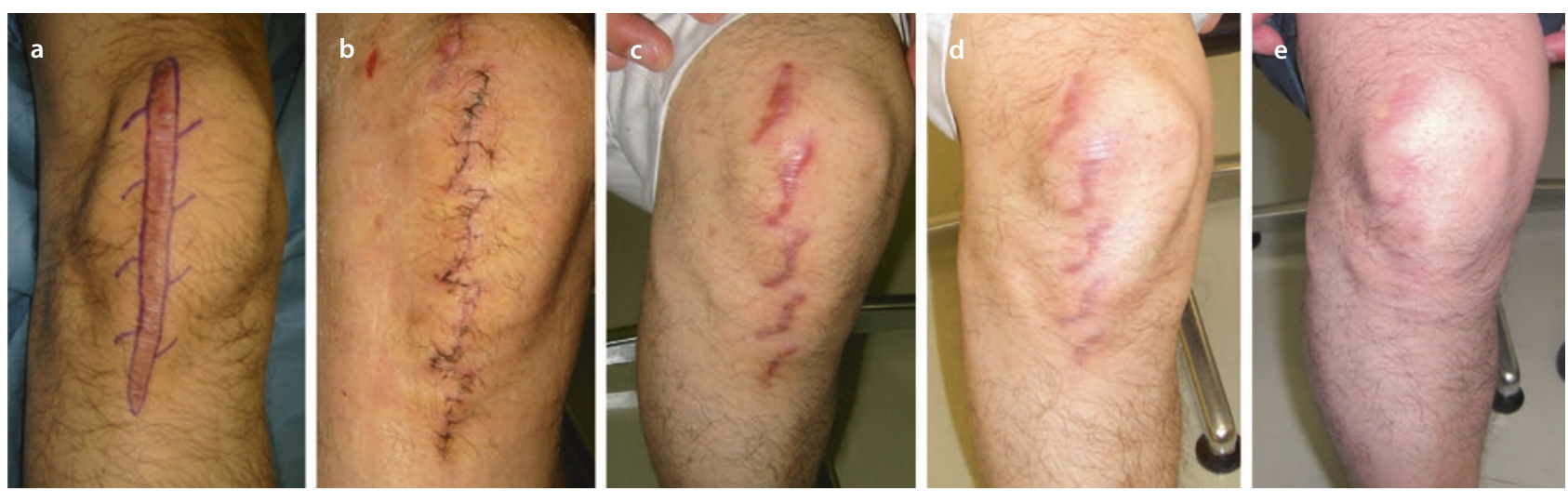

- Fig. 21.4 Knee joint scar revision. a Preoperative appearance of the scar and the design of the incisions and Z-plasties. b View immediately after the operation. c View 3 months after surgery. d View 6 months after surgery. e View 1 year after surgery. In the case of limb surgery, it is difficult to place deep tissue sutures. Therefore, it is

necessary to dissipate the tension on the wound by using zigzag line suturing methods such as the Z-plasty: this approach segments the scar and thereby releases its tension. This in turn causes the chronic tension-induced inflammation that is driving pathological scar growth to wane over time 

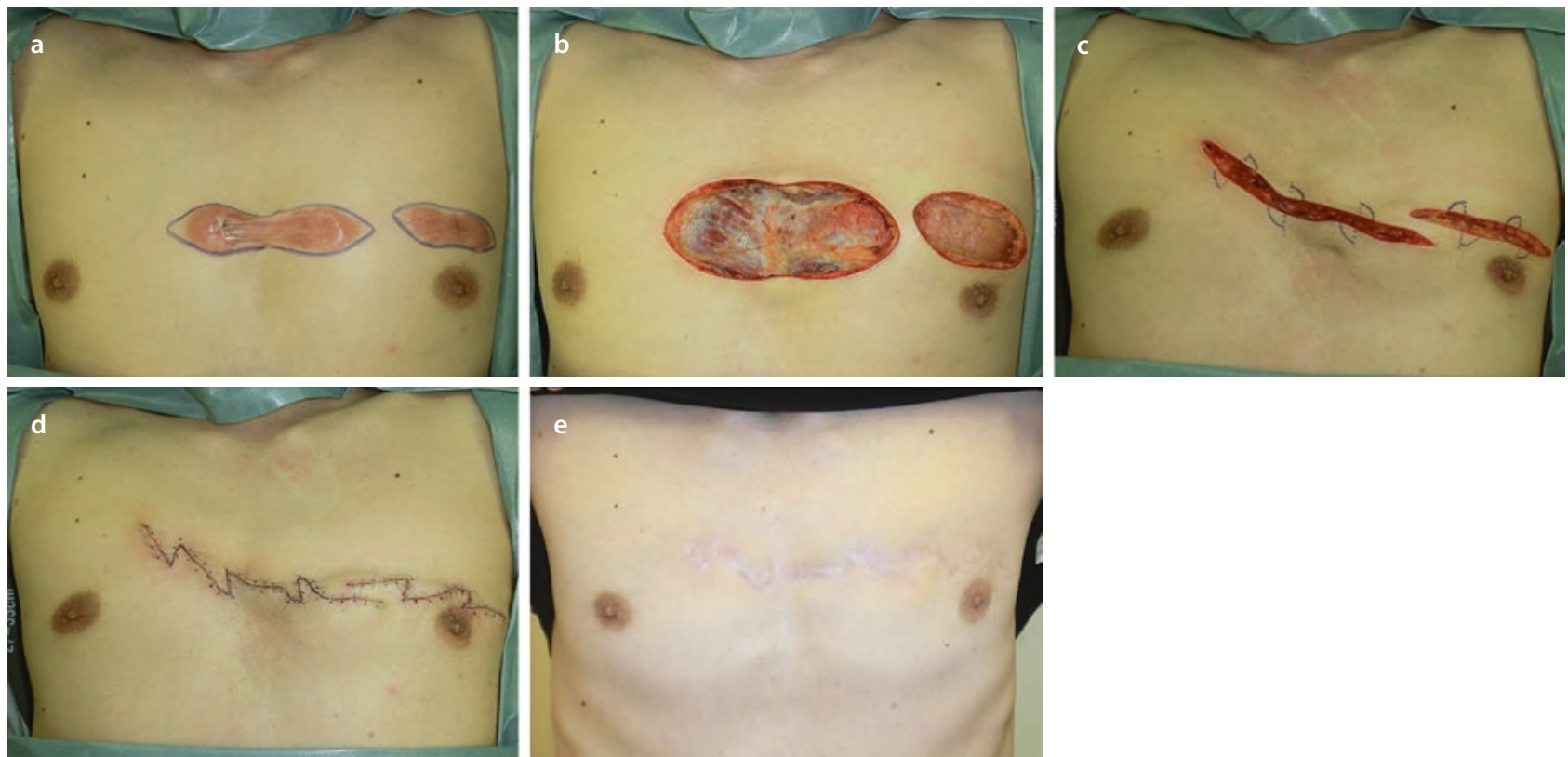

- Fig. 21.5 Anterior chest keloid treatment using tension reduction sutures, Z-plasties, and postoperative radiotherapy. a Preoperative appearance of the keloid and the design of the incisions. b View after the scar and fatty tissues were removed. c View immediately after the deep and superficial fascial sutures were placed and the Z-plasties were designed. d View immediately after the dermal

sutures and superficial sutures were placed. e View 2 years after surgery. In cases of keloid revision surgery, great care is needed to decrease the powerful chronic inflammation that is driving the growth of the scar. A combination of tension reduction sutures, Z-plasty, and postoperative radiotherapy can effectively prevent keloid recurrence after revision surgery

\subsection{Conclusion}

All four phases of wound healing are influenced by mechanical forces. These mechanical forces provoke chronic inflammation of the dermis and cause pathological scars. Dermal sutures do not effectively reduce tension on the dermis: rather, to achieve this, we must access much deeper structures, namely, the superficial and deep fascia, and suture them. Another way to prevent pathological scar formation in high-tension areas is to use zigzag suturing techniques such as the Z-plasty.

\section{Take-Home Messages}

- The risk of pathological scarring can be greatly reduced by using subcutaneous/fascial tensile reduction sutures.

- Dermal sutures do not effectively reduce tension on the dermis: rather, to achieve this, we must access much deeper structures, namely, the superficial and deep fascia, and suture them.

- A study showed that deep fascia suturing reduced about $90 \%$ of the tension on the wound edge, while superficial fascia suturing reduced the remaining $10 \%$.

- Z-plasty is particularly suitable for joint or limb surgery because the fatty tissues layers in these areas are thin: this means that it is difficult to find the superficial fascia and apply the subcutaneous/ fascial tensile reduction sutures. 


\section{References}

1. Levenson SM, Geever EF, Crowley LV, Oates JF III, Berard CW, Rosen H. The healing of rat skin wounds. Ann Surg. 1965;161:293-308.

2. Ogawa R. Mechanobiology of scarring. Wound Repair Regen. 2011;19(Suppl 1):s2-9.

3. Harn HI, Ogawa R, Hsu CK, Hughes MW, Tang MJ, Chuong CM. The tension biology of wound healing. Exp Dermatol. 2017.
4. Ogawa R, Akaishi S, Huang C, Dohi T, Aoki M, Omori Y, Koike $\mathrm{S}$, Kobe K, Akimoto M, Hyakusoku H. Clinical applications of basic research that shows reducing skin tension could prevent and treat abnormal scarring: the importance of fascial/subcutaneous tensile reduction sutures and flap surgery for keloid and hypertrophic scar reconstruction. J Nippon Med Sch. 2011;78(2):68-76.

5. Arima J, Dohi T, Kuribayashi S, Akaishi S, Ogawa R. Z-plasty and postoperative radiation therapy for anterior chest wall keloids: an analysis of 141 patients. Plast Reconstr Surg Glob Open. IN PRESS 2019.

Open Access This chapter is licensed under the terms of the Creative Commons Attribution 4.0 International License (http://creativecommons. org/licenses/by/4.0/), which permits use, sharing, adaptation, distribution and reproduction in any medium or format, as long as you give appropriate credit to the original author(s) and the source, provide a link to the Creative Commons license and indicate if changes were made.

The images or other third party material in this chapter are included in the chapter's Creative Commons license, unless indicated otherwise in a credit line to the material. If material is not included in the chapter's Creative Commons license and your intended use is not permitted by statutory regulation or exceeds the permitted use, you will need to obtain permission directly from the copyright holder. 